\title{
On-Board Generation of Three-Dimensional Constrained Entry Trajectories
}

\author{
Zuojun Shen* \\ Ping $\mathrm{Lu}^{\dagger}$ \\ Iowa State University \\ Ames, IA 50011-2271
}

Extended Abstract

A methododlogy for very fast design of 3DOF entry trajectories subject to all common inequality and equality constraints is developed. The approach make novel use of the wellknown quasi-equilibrium glide phenomenon in lifting entry as a center piece for convenirently enforcing the inequality constraints which are otherwise difficulty to handle. The algorithm is able to generate a complete feasible 3DOF entry trajectory, given the enttry conditions, values of constraint parameters, and final conditions in about 2 seconds on a PC. Numerical simulations with the X-33 vehicle model for various entry missions to land at Kennedy Space Center will be presented.

\section{Entry Guidance Problem Formulation}

The 3DOF equations of motion of the RLV over a spherical Earth in an Earth-centered and Earth-fixed coordinate system are given by ${ }^{1}$

$$
\begin{aligned}
\dot{r} & =V \sin \gamma \\
\dot{\theta} & =\frac{V \cos \gamma \sin \psi}{r \cos \phi} \\
\dot{\phi} & =\frac{V \cos \gamma \cos \psi}{r} \\
\dot{V} & =-D-\frac{\mu}{r^{2}} \sin \gamma \\
\dot{\gamma} & =\frac{1}{V}\left[L \cos \sigma+\left(V^{2}-\frac{\mu}{r}\right) \frac{\cos \gamma}{r}\right] \\
\dot{\psi} & =\frac{1}{V}\left(\frac{L \sin \sigma}{\cos \gamma}+\frac{V^{2}}{r} \cos \gamma \sin \psi \tan \phi\right)
\end{aligned}
$$

where $r$ the radial distance from the center of the earth to the vehicle. The longitude and latitude are $\theta$ and $\phi$, respectively. The Earth-relative velocity is $V$, and $D=\rho V^{2} S_{\text {ref }} C_{D} / 2 m$

\footnotetext{
-Graduate Research Assistant, Department of Aerospace Engineering and Engineering Mechanics

${ }_{\text {Associate Professor, Department of Aerospace Engineering and Engineering Mechanics, Associate Fellow }}$ AIAA. Emuil: plu@iustate.edu
} 
and $L=\rho V^{2} S_{\text {ref }} C_{L} / 2 m$ are aerodynamic drag and lift accelerations, where $S_{\text {ref }}$ is the reference area of the RLV, $m$ the mass, and $\rho$ the atmospheric density. The lift and drag coefficients $C_{L}$ and $C_{D}$ are modeled as functions of angle of attack $\alpha$ and Mach number. The atmospheric density $\rho$ can be approximated by an exponential function of the altitude $\rho=\rho_{0} e^{-\beta\left(r-R_{0}\right)}$, with $R_{0}=6378135 \mathrm{~m}, \beta>0$ and $\rho_{0}>0$ being constants. The Earth-relative flight path angle is $\gamma$ and $\sigma$ the bank angle. The velocity azimuth angle $\psi$ is measured from the North in a clockwise direction. Note that while the rotation of the Earth is important in simulating the trajectory of the RLV, it may be safely ignored in the entry trajectory planning and entry guidance law development. The feedback nature of the closed-loop entry guidance will easily compensate for the difference.

The entry guidance problem is defined as follows: given the entry conditions at the entry interface, the coordinates of the landing site, find the required bank angle profile $\sigma(t)$ and angle of attack history $\alpha(t)$ such that

1. The RLV reaches the terminal area energy management (TAEM) interface with specified conditions on altitude, velocity, velocity azimuth, and range to the heading alignment cone (HAC);

2. The trajectory observes path constraints that specify, respectively, maximum heating, load/acceleration, dynamic pressure, and equilibrium glide condition;

3. The $\alpha$-profile is consistent with the RLV trim capability, and both $\sigma$ and $\alpha$ do not exceed the flight control system authority. The latter constrains the maximum magnitude, rate and acceleration of $\sigma$ and $\alpha$.

The entry guidance problem is traditionally addressed in two parts:

(1) off-line design of a reference: This is where some type of reference trajectory/profile is iteratively designed to meet the above requirements;

(2) on-board feedback tracking of the reference and minor adjustments when necessary.

The capability of generating a feasible reference trajectory on-board will provide great flexibility to the RLV operation and eliminate a most labor-intensive part from the entry guidance planning. The two key ingredients for a fully automated entry guidance system are on-board generation of a reference trajectory and a tracking control law that automatically adjust to the trajectory generated (no gain-scheduling needed). The second task has been successfully addressed by a recent development. ${ }^{2}$ The first task is addressed here.

\section{Rapid Generation of 3DOF Entry Trajectories}

The chief challenges of on-board entry trajectory generation lie in designing the 3DOF trajectory to meet all the path (inequality) constraints reliably in all cases. Even in off-line trajectory planning, the commonly employed methods for trajectory design will inevitably require many iterations. Often times it takes experience, good understanding of the method used, and insight into the mechanics of the entry flight to "tweak" the process to get satisfactory results. In other attempts to generate the entry trajectory on-board (e.g., $\left.{ }^{3}\right)$, the 
path constraints are not considered at all. Without the ability to ensure path constraints not to be violated, the usefulness of such an algorithm would be very limited. Since the dynamics of the RLV in Eqs. (1-6) are highly nonlinear and lack any special features such as differential flatness, the success of an on-board trajectory generation algorithm relies on cleverly decomposing this infinite-dimensional search problem into a finite-dimensional search problem with a very small dimension.

\subsection{Assumptions and Objective}

We shall refer to a solution of the differential equations (1-6) (state and inputs $\sigma(t)$ and $\alpha(t))$ subject to all the imposed conditions listed in Section 1 for a given RLV as a feasible trajectory for the RLV. The following assumptions establish the scope of applicability of the algorithms to be presented.

1. The entry flight of the RLV is lifting entry $(L / D \neq 0)$.

2. The TAEM conditions are specified in terms of altitude, velocity, range-to-HAC, and velocity heading pointing to the HAC.

3. All the path constraints can be expressed as constraints in the altitude and velocity space (when $\alpha$ is given).

4. There exists at least one feasible trajectory for the given entry conditions, imposed TAEM conditions, path constraints, and the given vehicle capability.

5. A nominal $\alpha$-versus-Mach profile is available, and limited variations about this nominal profile are allowable.

The last assumption is not a necessity for the algorithms, but a practical condition. An arbitrarily designed $\alpha(t)$ profile may not be compatible with the requirements due to thermal protection and flight trim conditions. And a nominal $\alpha$ profile, once determined for a given vehicle, typically does not change significantly from mission to mission. The Assumption 3 encompasses all the typical constraints considered in entry flight.

The objective is to find a feasible 3DOF trajectory within a short time period that is comparable to on-board guidance environment. Such an algorithm not only can be applied on-board, but will also provides a fast tool for off-line trajectory generation and analysis. It should be noted that no optimization of any particular performance index is explicitly attempted in the design. But for entry flight, the trajectory is highly constrained by the path cosntraints and TAEM conditions so that an optimal and a feasible trajectory do not differ appreciably.

\subsection{Quasi-Equilibrium Glide Condition}

The centerpiece in this algorithm for path-constraint enforcement is the use of the quasiequilibrium glide condition. It is well known in flight mechanics that along a major portion of a lifting entry trajectory, the flight path angle $\gamma$ is small and varies relatively slowly. For 
a constant bank angle $\sigma$, setting $\cos \gamma=1$ and $\dot{\gamma}=0$ in Eq. (5) produces the so-called equilibrium glide condition

$$
L \cos \sigma+\left(V^{2}-\frac{\mu}{r}\right) \frac{1}{r}=0
$$

In actual flight, $\sigma$ is not a constant, but the above observations are still true. In other words, for a major portion of the lifting entry flight, at any point of the trajectory where the pair $(r(t), V(t))$ is given, the value of the bank angle at that point will be close to the corresponding value of $\sigma(t)$ from condition (7). Clearly the value of $\sigma$ from Eq. (7) is generally different from one point to another along the trajectory. Henceforth we shall call the condition (7) the quasi-equilibrium glide condition (QEGC) to signify that we allow time-varying $\sigma$ in Eq. (7).

The QEGC provides us a simple and effective means to shape the altitude and velocity profiles. For any given value of $V$ at a point of the trajectory, if a value of $\sigma$ is selected, the value of altitude at that point is determined from the QEGC. If $\sigma$ is selected between a maximum and minimum value, the corresponding altitude will be between a minimum and maximum range. The obvious lower bound for $\sigma$ in this process is zero degree, or a fixed value when the equilibrium glide condition is desired to be enforced at that value. The maximum value of $\sigma$ is determined in the following way: Suppose that the path constraints are

$$
s_{i}(r, V, \alpha) \leq c_{i}, \quad i=1, \ldots, l
$$

where $c_{i} \geq 0$ is constant. The region in the $r-V$ space where all the above conditions are met is called the entry flight corridor. For all the commonly used entry path constraints, the value of $s_{i}(r, V, \alpha)$ increase as the altitude decreases (atmospheric density increases). Figure 1 shows the nominal entry trajectory for the X-38 Crew Return Vehicle and several typical path constraints. 


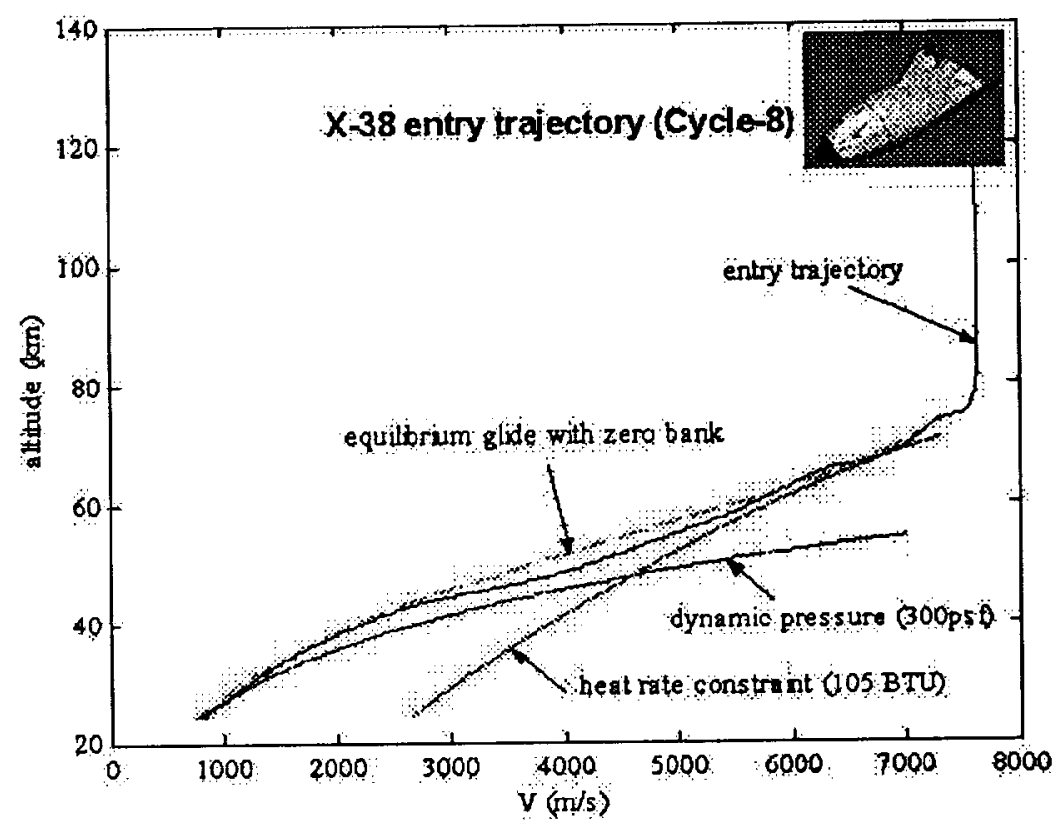

Fig. 1: Entry trajectory for the X-38 Crew Return Vehicle

On the constraint boundary $s_{i}(r, V, \alpha)=c_{i}$, for any given $V$, the value of $r$ is determined because $\alpha$ is from the nominal $\alpha$-versus-Mach profile. This $r$ represents the lowest altitude at this velocity without violating the $i$-th constraint. Find the corresponding bank angle from the QEGC, and denote it by $\sigma_{\max }^{i}(V)$ because it will be the maximum allowable bank angle at this velocity without violating the constraint $s_{i}(r, V, \alpha) \leq c_{i}$. Define for all $V$ in the portion of the entry trajectory where the QEGC is valid

$$
\sigma_{\max }(V)=\min _{1 \leq i \leq l}\left\{\sigma_{\max }^{i}(V)\right\}
$$

Then, for any such $V$, when we choose a $\sigma \leq \sigma_{\max }(V)$, the corresponding $r$ from the QEGC will ensure that all the path constraints in Eq. (8) are satisfied. Figure 2 shows the variation of such $\sigma_{\max }(V)$ for the X-38 and the corresponding boundaries of the entry flight corridor. 

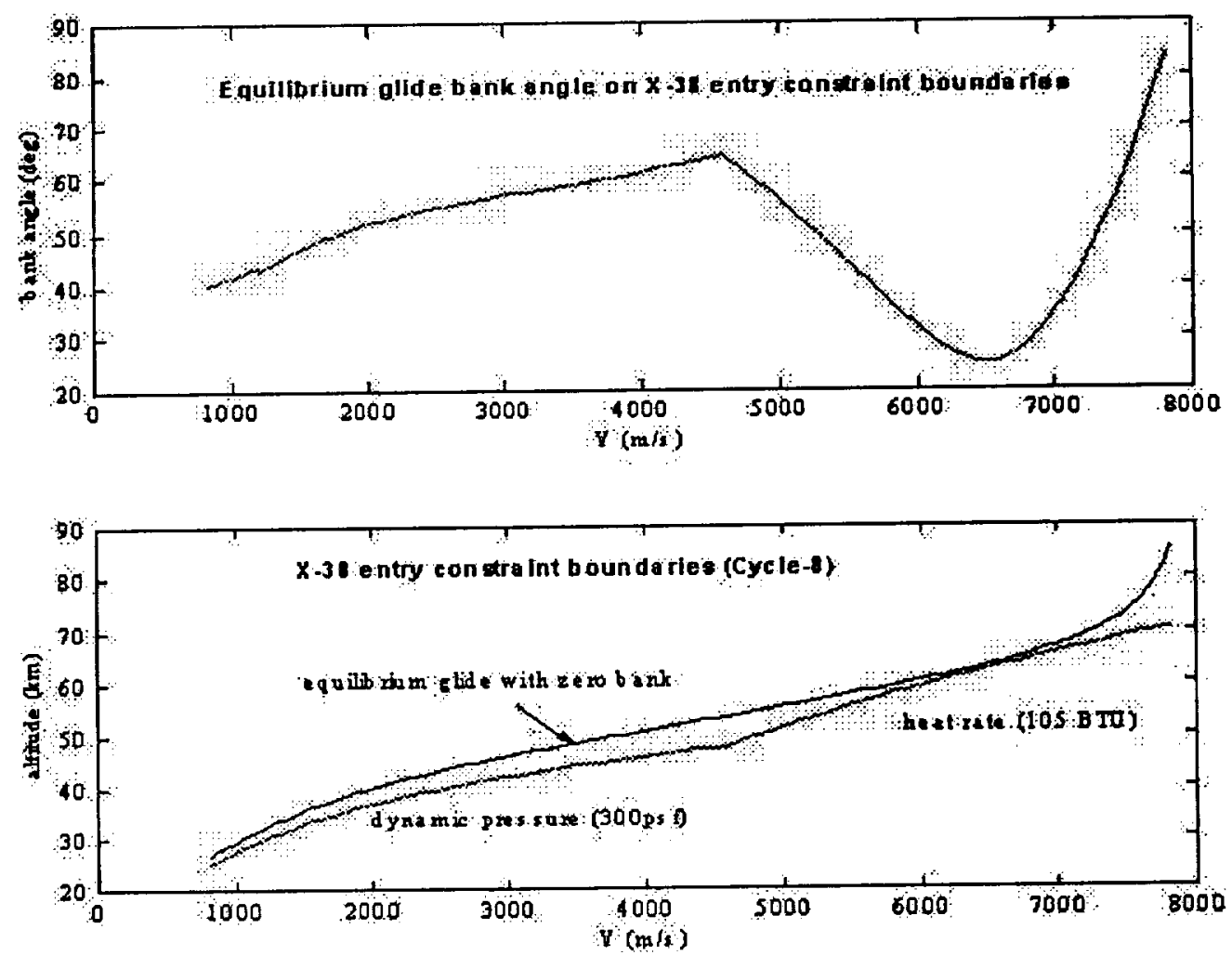

Fig. 2: X-38 Entry flight corridor and $\sigma$ from QEGC for the path constraints

\subsection{Altitude- and Velocity-versus-Range Profiles}

The QEGC will be used to facilitate the search for $r$ - and $V$-versus-range profiles satisfying path and TAEM constraints into a one-parameter search. All the steps described hereafter are automated. Note that the QEGC can only be satisfied by some triplet $(r, V, \sigma)$ once sufficient dynamic pressure has been built up. For orbital entry, there will be an initial period of the flight where the atmospheric density is too low for the QEGC to be met by any triplet $(r, V, \sigma)$. In such a case in the trajectory design process we start with an initial descent phase where $\sigma=\sigma_{0}$, a constant.

\section{Initial Descent}

The constant $\sigma_{0}$ is chosen such that the initial descent will enter the entry flight corridor and transition smoothly onto the QEGC. Since the heading of the RLV at entry interface is pointed toward the landing site, the differential equation for the range-to-go to the HAC may be written as

$$
\dot{s}_{\text {togo }}=-\frac{V \cos \gamma}{r}
$$


Using $s_{\text {togo }}$ as the independent variable, and for the chosen $\sigma_{0}$ and the nominal $\alpha$-profile, the Eqs. (1), (4) and (5) are numerically integrated. The QEGC is continuously checked during the integration. Once the QEGC begins to admit a solution $\sigma$ for the pair $(r, V)$ obtained, we start the checking for transition point onto the QEGC. The most effective criterion for determining the transition point we found is to compare the slope of $\partial V / \partial r$ on the numerically integrated descent portion with that along the QEGC. Whenever the two slopes match within a tolerance, the trajectory transitions onto the QEGC.

The value of $\sigma_{0}$ is first set to be zero. But in some cases $\sigma_{0}=0$ will result in the trajectory bouncing a few times before it can transitions onto the QEGC (characterized by large oscillations in the altitude history, a known phenomenon in entry flight). If this undesirable bouncing happens, a new $\sigma_{0}>0$ is selected in a pre-selected increment and the numerical integration restarted from the entry interface. Note that the path constraints are all inactive in this phase because the atmospheric density is still very low. At most 3-4 repetitions, this process will have been completed, and the trajectory is on the QEGC. The integrated $r\left(s_{\text {togo }}\right)$ and $V\left(s_{\text {togo }}\right)$ are stored for later use.

\section{Determination of Bank Angle Profile on QEGC}

The values of $\sigma$ at the first point and the last (the TAEM point) on the QEGC are solved Eq. (7), since the values of $r$ and $V$ are already known or specified. Denote them by $\sigma_{1}$ and $\sigma_{T A E M}$. Let $\sigma_{m i d}$ be a value to be determined at the midway point of the TAEM velocity and $V_{1}$, the velocity at the transition point from the initial descent to QEGC. A piecewise linear, continuous $\sigma(V)$ profile can be obtained using $\sigma_{1}, \sigma_{m i d}$ and $\sigma_{T A E M}$ (or a quadratic $\sigma(V)$ if an additional condition $d \sigma(V) / d V=0$ at $\sigma_{\text {mid }}$ is used). Again in Eq. (4), use $s_{\text {togo }}$ as the independent variable, $\sin \gamma \approx 0, \cos \gamma \approx 1$, and replace $D=\left(C_{D} / C_{L}\right) L$ where $L$ is substituted from the QEGC (7)

$$
\frac{d V}{d s_{\text {togo }}}=\left(\frac{\mu}{r}-V^{2}\right) \frac{\left(C_{D} / C_{L}\right)}{V \cos \sigma}
$$

For any selected $\sigma_{m i d}, \sigma(V)$ is determined for all $V \in\left[V_{T A E M}, V_{1}\right]$. At any $V$ and the corresponding $\sigma(V)$, the value of $r$ is found from the QEGC. From the nominal $\alpha$ profile, and the given RLV aerodynamic model, $C_{D} / C_{L}$ is evaluated. Thus Eq. (11) can be numerically integrated from the $s_{\text {togo }}$ at the transition point onto the QEGC to the required range-to-HAC at the TAEM interface. The resulting TAEM velocity is obtained. If the resulting TAEM velocity is not equal to the required $V_{T A E M}, \sigma_{m i d}$ is adjusted and the process repeats. The corresponding $r$ profile is also obtained by evaluating the value of $r$ from the QEGC at any integrated velocity value. Note that the $r$-versus-range profile obtained this way satisfies the required TAEM altitude condition automatically if the TAEM velocity is equal to the required value, because the last point on the $\sigma(V)$ profile, $\sigma_{T A E M}$, is calculated from the QEGC by using the required values of $r$ and $V$ at TAEM.

The variation of the velocity at the TAEM is monotonic with respect to $\sigma_{\text {mid }}$ because larger value of $\sigma_{\text {mid }}$ means larger $\sigma(V)$ at any given $V$, hence smaller velocity at TAEM (due to faster energy dissipation); the opposite is also true. This monotonic functional behavior renders that the search for $\sigma_{m i d}$ to achieve required TAEM velocity will converge reliably 
and quickly with a simple secant algorithm: Let $V_{\text {TAEM }}$ be the required TAEM velocity, and $V_{\text {TAEM }}^{i}$ the velocity at TAEM in the $i$-th search, corresponding to $\sigma_{\text {mid }}^{i}$. Then the secant algorithm is

$$
\sigma_{m i d}^{i+1}=\sigma_{m i d}^{i}-\frac{\sigma_{m i d}^{i}-\sigma_{m i d}^{i-1}}{V_{T A E M}^{i}-V_{T A E M}^{i-1}}\left(V_{T A E M}^{i}-V_{T A E M}\right)
$$

Note for each $\sigma_{m i d}^{i}$ a bank angle profile $\sigma(V)$ is defined, and Eq. (11) is integrated to get $V_{T A E M}^{i}$. In this process, the path constraints are enforced by imposing on $\sigma(V)$ the condition

$$
\sigma_{\min } \leq \sigma(V) \leq \sigma_{\max }(V)
$$

where $\sigma_{\max }(V)$ is found in Eq. (9), and $\sigma_{\min } \geq 0$ is a constant if an equilibrium glide condition at that constant bank angle is also desired to be enforced as in some cases. ${ }^{4}$ The $r$ - and $V$-versus-range profiles obtained will ensure that all the path constraints, range-toHAC condition, and TAEM conditions on $r$ and $V$ are met. The QEGC not only enables us to reduce the need to numerically integrate more equations thus reduce the computation time, more importantly it allows us to enforce the path constraints so reliably and effortlessly.

Once the above one-parameter search is completed, the obtained $r$ and $V$ profiles are appended to these obtained for the initial descent. Now we have $r$ and $V$ versus range-to-go profiles from entry to TAEM, and the magnitude of $\sigma$ along these profiles.

\subsection{Completion of 3DOF Trajectory}

Thus far the longitudinal state $r$ and $V$ versus range-to-go, and the magnitude of the bank angle have been determined. To complete the 3DOF trajectory design, the sign of $\sigma$ and the rest of the state need to be determined. This task is accomplished by tracking the obtained $r$ and $V$ versus range-to-go profiles with linear time-varying control laws for $\sigma$ and $\alpha .^{2}$ From the $r$ and $V$ profiles, the corresponding $\gamma$ is easily backed out from Eq. (1). Note that the longitudinal dynamics Eqs. (1), (4) and (5) are decoupled from the rest of the state. Use the just obtained $r, V, \gamma, \sigma$ (magnitude), and nominal $\alpha$ profile as the references. $\mathbf{x}_{l o n}=(r V \gamma)^{T}$ and $\mathbf{u}=(\sigma \alpha)^{T}$. Linearize the longitudinal dynamics, and still use range-to-go $s_{\text {togo }}$ as the independent variable. Let the linearized longitudinal dynamics be

$$
\dot{\mathbf{x}}_{\text {lon }}=A\left(s_{\text {togo }}\right) \delta \mathbf{x}_{\text {lon }}+B\left(s_{\text {togo }}\right) \delta \mathbf{u}
$$

The trajectory control law developed in Ref. $^{2}$ will lead to

$$
\delta \mathrm{u}=-K\left(s_{\text {togo }}\right) \delta \mathbf{x}_{\text {lon }}
$$

where the feedback gain $K\left(s_{\text {togo }}\right)$ is a function of $A$ and $B$ in Eq. (14) in closed-form. The modified bank angle $\sigma$ and angle of attack will be

$$
\mathbf{u}=\mathbf{u}_{r e f}+\delta \mathbf{u}
$$


where $u_{\text {ref }}$ represent the bank angle profile obtained in preceding sections and nominal $\alpha$. These controls in (16) are used to numerically integrate the complete 3DOF nonlinear dynamics (1-6) from the entry interface. The range-to-go used to schedule the control law (15) is the actual range-to-go found from the integrated values of longitude $\theta$ and latitude $\phi$. The initial sign of $\sigma$ is set to be the opposite of the sign of $\Delta \psi_{H A C}=\left(\psi-\Psi_{H A C}\right)$, where $\Psi_{H A C}$ is the azimuth angle to the HAC. The sign of $\sigma$ is reversed at a range $s_{\text {rev }}$ that is to be determined iteratively. The criterion for determining $s_{\text {rev }}$ is that $\triangle \psi_{H A C}$ at TAEM interface falls within the acceptable range (e.g., $\pm 5 \mathrm{deg}$ ). Again the secant method appears to work well for this search. This search involves integration of all the dynamic equations (1-6), but does not need to be from the very beginning (entry interface).

During this process, any limits on the magnitude of variations, rate and acceleration of the controls $\sigma$ and $\alpha$ can be applied. Once the integration (and search for $s_{\text {rev }}$ ) is completed, the 3DOF state trajectory and corresponding bank angle and angle of attack are obtained and stored as the reference trajectory.

Compared to current standards in off-line trajectory planning, the above described trajectory generation algorithms produce a feasible trajectory very rapidly (about 2 seconds from a cold start, on a PC with a $800 \mathrm{MHZ}$ processor). There are no apparent benefits in entry flight to require the generation of the reference trajectory in every guidance cycle. Instead, The generated reference trajectory will be tracked by another guidance law similar to (15), but is based on the linearized dynamics of the overall system, not just the longitudinal dynamics. This guidance law will track both longitudinal and lateral state variables, and generate the actual guidance commands as described in Ref. ${ }^{2}$ If necessary, the trajectory generation algorithms may be called again when the deviations from the reference trajectory become too large. In such a case the current condition of the RLV is used as the initial condition, and a new feasible reference trajectory is generated starting from the current state.

\section{Summary of the Algorithms}

The following is a re-cap of the steps described in the preceding sections for the trajectory generation algorithms:

1. An initial descent phase when entry from orbit till sufficient dynamic pressure has been built up for transition to the QEGC. A constant bank angle is determined in this phase to ensure desirable descent behavior. The transition point is also found which marks the end of the initial descent phase.

2. A single parameter search defining the bank angle profile is done by using the QEGC and numerically integrating the velocity equation. This process determines the altitudeand velocity-versus-range profiles that satisfy all the path constraints and TAEM conditions on range-to-HAC, altitude and velocity. The magnitude of the bank angle along the QEGC is also found.

3. Reduced order linear time-varying control laws for bank angle and $\alpha$ are employed to track the just obtained altitude and velocity profiles. The complete set of 3DOF 
dynamic equations are numerically integrated using the control law outputs. The sign of the bank angle is determined by a single bank reversal. The range at which the bank reversal occurs is iteratively determined by a secant method to reduce the heading-to-HAC error at the TAEM interface within the targeting limit. The final 3DOF state trajectory and corresponding bank angle and angle of attack profiles are stored on-board as the reference trajectory.

4. Linear time-varying trajectory control laws for $\sigma$ and $\alpha$ based on the full-order 3DOF RLV dynamics are used on-line to track the reference trajectory. The actual guidance commands are the combinations of the reference bank angle and angle of attack and the outputs of these control laws.

5. When necessary, new reference trajectory may be generated mid-stream by calling the algorithms again.

\section{Partial References}

1 Vinh, N. X., et al., Hypersonic and Planetary Entry Flight Mechanics, The University of Michigan Press, Ann Arbor, MI, 1980.

2 Lu, P., "Regulation about Time-Varying Trajectories: Precision Entry Guidance Illustrated", Journal of Guidance, Control, and Dynamics, Vol. 22, No. 6, 1999, pp. 784-790.

3 Fuhry, D. P., "Adaptive Atmospheric Reentry Guidance for the Kistler K-1 Orbital Vehicle", Proceedings of AIAA Guidance, Navigation, and Control Conference, Denver, $\mathrm{CO}$, August 14-17, 2000

4 Tiggs, M. A., Preliminary V-201 Deorbit/Entry Flight Profile with Landing Site Precision, Cycle 9, NOI\# 314, NASA Johnson Space Center, February 1, 2001. 